\title{
Early Second-Trimester Peptidomic Identification of Serum Peptides for Potential Prediction of Gestational Diabetes Mellitus
}

\author{
Lingfeng Yin ${ }^{\mathrm{a}}$ Yingying Huaib ${ }^{\mathrm{b}}$ Chun Zhao ${ }^{\mathrm{a}}$ Hongjuan Ding ${ }^{\mathrm{a}}$ \\ Tao Jiang $^{a} \quad$ Zhonghua Shi ${ }^{a}$ \\ aState Key Laboratory of Reproductive Medicine, Women's Hospital of Nanjing Medical University,

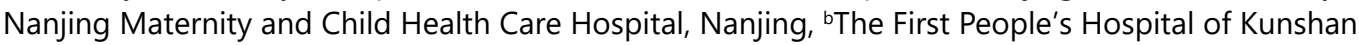 \\ Affiliated to Jiangsu University, Kunshan, China
}

\section{Key Words}

GDM • Peptide $\cdot$ Serum $\cdot$ Prediction

\begin{abstract}
Background/Aims: Early screening and diagnosis is important for minimizing gestational adverse outcomes. Routine screening of gestational diabetes mellitus (GDM) at 24-28 weeks with $75 \mathrm{~g}$ oral glucose challenge test (OGCT) leaves limited time for intervention and prevention. This study aims to analyze maternal serum peptides in the early second-trimester for prediction of gestational diabetes mellitus (GDM). Methods: Serum samples were collected from 16-18-week pregnant women that visited Nanjing Maternity and Child Health Care Hospital from April to August 2015. According to gestational outcome with or without GDM in late pregnancy, 200 of serum samples from GDM mothers and controls were randomly divided into two subgroups. Peptidomic identification of serum peptides was performed by combining ultrafiltration and liquid chromatography-tandem mass spectrometry (LC-MS/MS) to investigate the differentially-expressed peptides between two groups. Results: A total of 297 identified peptides, originating from 228 proteins, were significantly differentially expressed in the GDM group compared with control. These precursor proteins may play critical roles in cell death of cortical neurons, elongation of cellular protrusions, and stabilization of microtubules. Major networks identified included those involving lipid metabolism, molecular transport and small molecule biochemistry. Conclusion: We provide for the first time a validated peptidome profile of early second-trimester serum in normal and GDM mothers, and we investigated the potential serum biomarkers for GDM. We concluded that 297 peptides could serve as potential biomarkers for GDM.

L. Yin, Y. Huai and C. Zhao contributed equally to this work. 


\section{Cellular Physiology Cell Physiol Biochem 2018;51:1264-1275

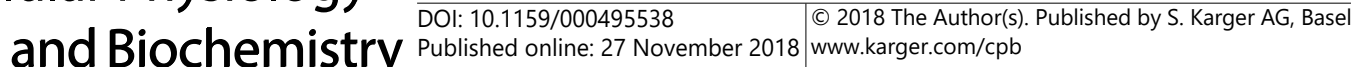 \\ Yin et al.: Peptidomic Identification for Predicting Gestational Diabetes Mellitus}

\section{Introduction}

Gestational diabetes mellitus (GDM) is defined as a condition in which carbohydrate intolerance develops during pregnancy [1]. As rates of obesity and diabetes have increased rapidly in recent years, the prevalence of GDM is also increasing and is now estimated at $1.7 \%-11.6 \%[2,3]$. The main adverse impact of GDM on pregnancy includes pregnancyinduced hypertension, fetal macrosomia, shoulder dystocia or birth injury, and premature delivery. Evidence also exists that women diagnosed with GDM in pregnancy are at a higher future risk of developing type 2 diabetes, metabolic complications, and cardiovascular diseases [4-6].

Currently, there is no consensus as to the ideal screening method for GDM. The two competing approaches are the "2-step and the "1-step" approaches. The 2-step approach begins at about 24 to 28 weeks' gestation with an oral glucose challenge test (OGCT). This is followed by a 3-hour OGTT. The 1-step approach consists of a 2-hour OGTT only. Screening method and prevalence of GDM varies by geography. Body mass index (BMI), maternal age, and certain features of medical history are measures that must be changed with risk factors. However, all these measures are relatively weak as predictors [7, 8]. A method that can more effectively select women at high risk of GDM would be beneficial, especially if it was implemented early in pregnancy. Early intervention, such as diet, medication, and exercise, could avoid the development of GDM or avoid adverse maternal and fetal sequelae. To that end, we examined potential first-trimester serum markers for the prediction of subsequent GDM.

An area of increasing interest in the field of biomarker discovery is peptidomics, defined as the systematic qualitative and quantitative analysis of peptides in biological samples [911]. Evidence is accumulating to suggest that endogenous peptides play critical roles in the cardiovascular, endocrine, nervous, respiratory, inflammatory and reproductive systems [12, 13]. However, few studies have investigated the relationship between peptidome profiles in serum and early diagnosis of GDM.

Serum peptides can act as biomarkers, providing valuable information regarding diagnoses and disease states. In this study, we test the hypothesis that serum peptides might serve as potential biomarkers for predicting GDM in early pregnancy.

\section{Materials and Methods}

\section{Ethics Statement}

This study was approved by the Ethics Committee of the Gynecology and Obstetrics Hospital Affiliated to Nanjing Medical University (ethics committee approval number: NJFY-2015-18) and conducted in accordance with the Declaration of Helsinki. All subjects gave written informed consent prior to the start of the study. Sample collection was performed in accordance with the ethical standards of the responsible committee on human experimentation (institutional and national).

\section{Subjects}

Serum samples were collected from 16- to 18-week pregnant women that visited the Nanjing Maternity and Child Health Care Hospital from April to August 2015. Clinical data and outcomes of GDM women and their paired controls were collected until delivery. All study subjects were healthy and showed no significant differences in age, body mass index (BMI), ethnicity, and gestational stage at the time of blood collection (Table 1). Exclusion criteria for both groups were as follows: hypertensive disorders; history of diabetes prior to conception; obesity; smoking; chemical dependency; use of assisted reproductive technology; multiple gestation; fetal congenital anomalies; and any other confounding pathologies, including hyperthyroidism and hypothyroidism.

Subjects were divided into two groups based on the diagnostic criteria of OGTT recommended by the International Association of the Diabetes in Pregnancy Study Group (IADPSG) at 24-28 weeks: healthy pregnant women and patients with GDM. GDM was diagnosed if fasting glucose $\geq 5.1 \mathrm{mmol} / \mathrm{L}$, or if the 1 


\section{Cellular Physiology Cell Physiol Biochem 2018;51:1264-1275

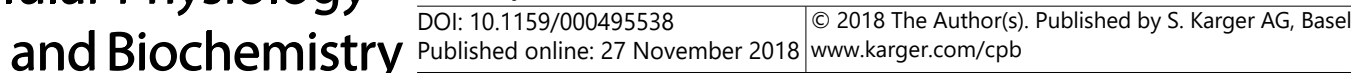 \\ Yin et al.: Peptidomic Identification for Predicting Gestational Diabetes Mellitus}

$\mathrm{h}$ result $\geq 10.0 \mathrm{mmol} / \mathrm{L}$ or the $2 \mathrm{~h}$ result $\geq$ $8.5 \mathrm{mmol} / \mathrm{L}$. The remaining women were placed in the healthy group.

Serum sample collection, preparation, and ultrafiltration

Blood samples were obtained at 16 to 18 weeks of gestation at the time of screening for Down's syndrome. Samples were centrifuged at $4000 \mathrm{rpm}$ at $4^{\circ} \mathrm{C}$ for $10 \mathrm{~min}$ to separate them into serum and cellular fractions, followed by centrifugation at $12000 \mathrm{rpm}$ for $15 \mathrm{~min}$ to completely remove cell debris.

After thawing on ice, the samples were centrifuged at $12000 \mathrm{rpm}$ for $15 \mathrm{~min}$ at $4^{\circ} \mathrm{C}$. Then, $20 \%(\mathrm{v} / \mathrm{v}$ ) Acetonitrile (CAN) was added to the supernatants, and the samples were briefly vortexed and incubated for $20 \mathrm{~min}$ at room temperature. Molecular weight cut-off (MWCO) filters (Millipore, USA) of $10 \mathrm{kDa}$ were washed with $0.5 \mathrm{~mL}$ water. The samples were passed through the filters according to the manufacturer's recommendations. The flow-through containing peptides was recovered and lyophilized. Protein concentrations were determined using the bicinchoninic acid method (BCA; Pierce, Rockford, IL, USA).

Peptide desalting and multiplexed isobaric tandem mass tag (TMT) labeling

Samples containing $100 \mu \mathrm{g}$ peptides were reduced with $10 \mathrm{mM}$ DTT at $60^{\circ} \mathrm{C}$ for $1 \mathrm{~h}$, alkylated with $55 \mathrm{mM}$ iodoacetamide (IAA) for $45 \mathrm{~min}$ at room temperature, then desalted and dried in vacuo (Speed Vac, Eppendorf). AF peptides were labeled with isobaric tags based on total peptide amount at room temperature for $1 \mathrm{~h}$. TMT labeling was performed according to manufacturer's instructions (TMT 6-plex Label Reagent, Thermo Scientific). TMT results were generated from analysis of isobaric tag combinations. Three normal control AF samples were labeled with reagents 126, 127 and 128, and three VSD AF samples were labeled with reagents 129,130 and 131.

\section{Mass spectrometry analysis, peptide identification and quantification}

Peptides were analyzed by nanoflow LC (Easy-nLC, Thermo

Fisher Scientific Inc., San Jose, CA) combined with an LTQ-Orbitrap Velos mass spectrometer (Thermo Fisher Scientific Inc.). Reverse-phase separation of peptides was performed using buffer A ( $2 \%$ ACN, $0.5 \%$ acetic acid) and buffer B ( $80 \%$ ACN, $0.5 \%$ acetic acid) with a gradient. Eluted peptides were electrosprayed at $1.8 \mathrm{kV}$ into the mass spectrometer which was configured to collect high resolution $(\mathrm{R}=60,000$ at $\mathrm{m} / \mathrm{z}$ 400) broadband mass spectra (m/z 375-1800) using the lock mass feature for the polydimethylcyclosiloxane ion generated in the electrospray process ( $\mathrm{m} / \mathrm{z} 445.12002)$. The 20 most abundant peptide molecular ions dynamically determined from the MS scan were selected for tandem MS using a relative collision-induced dissociation (CID) energy of 35\%. The most intense product ion from the MS2 step was selected for higher energy collision-induced dissociation (HCD) MS3 fragmentation.

Extracted MS/MS spectra were searched against the database containing 20, 194 human protein sequences (released May 2015) using in-house PEAKS software (version7.0, Bioinformatics Solutions, Waterloo, Canada). The fusion target-decoy approach was used for estimation of the false discovery rate (FDR) and was set at $\leq 1 \%(-10 \log \mathrm{P} \geq 20.0)$ at both protein and peptide levels. Peptides were considered positively identified only if peptide peaks were present in at least two spectra per sample.

Relative quantification of the serum peptidome was performed using the TMT labeling approach in the PEAKS Q module. Feature detection was performed separately on each sample using the expectationmaximization algorithm. Features of identical peptides from different samples were aligned using the high-performance retention time alignment algorithm [14]. Peptides and proteins were considered to be significantly altered between serum samples when the p-value was $<0.05$ and the fold change was $>1.5$. Identified results were included during the last step of TMT labeling quantification. 


\section{Bioinformatics analysis}

The isoelectric point (pI) of each peptide was calculated using the pI/Mw online tool (http://web. expasy.org/compute_pi/). Pathway and gene ontology (GO) analyses (http://geneontology.org) were carried to determine potential physiological functions. GO results included three categories; cellular component, biological process and molecular function. The threshold of significance was defined by the P-value and FDR. To further explore the significance of differentially-expressed peptides and their precursor proteins, they were imported into the Ingenuity Pathway Analysis (IPA) Software v7.1 (Ingenuity Systems, Mountain View, CA) for pathway analysis as described previously [15]. This software can also be used for biochemical, biologic, and molecular functions. The identified proteins were mapped to associated network functions that were generated from the Ingenuity Systems Knowledge Base. The MEROPS database (http://merops.sanger.ac.uk) was used to seek out candidate enzymes for given cleavage sites [16].

\section{Statistical Analysis}

SPSS 15.0 software (SPSS Inc, Chicago, IL). The t-test was used to compare values between control and GDM groups. A p value $<0.05$ was considered to indicate statistical significance.

\section{Results}

Identification of various peptides in serum

The peptides from serum were directly analyzed by LC-MS/ MS. A total of 297 peptides that were significantly differentially expressed (fold change > 1.5) are listed in Supplement 1. The molecular weight (MW) and isoelectric point (pI) distribution characteristics of these peptides are shown in Fig. 1. The MW vs. pI distribution of the identified peptides is shown in Fig. 1C. The MWs of all identified peptides were below $3 \mathrm{kDa}$, confirming that isolated peptides were of adequate purity.

\section{Bioinformatics analysis of precursors of differentially expressed peptides}

A total of 228 precursors of identified peptides were analysis using the PANTHER database to identify canonical pathways, diseases and biological functions and networks. A total of 37 statistically significant canonical

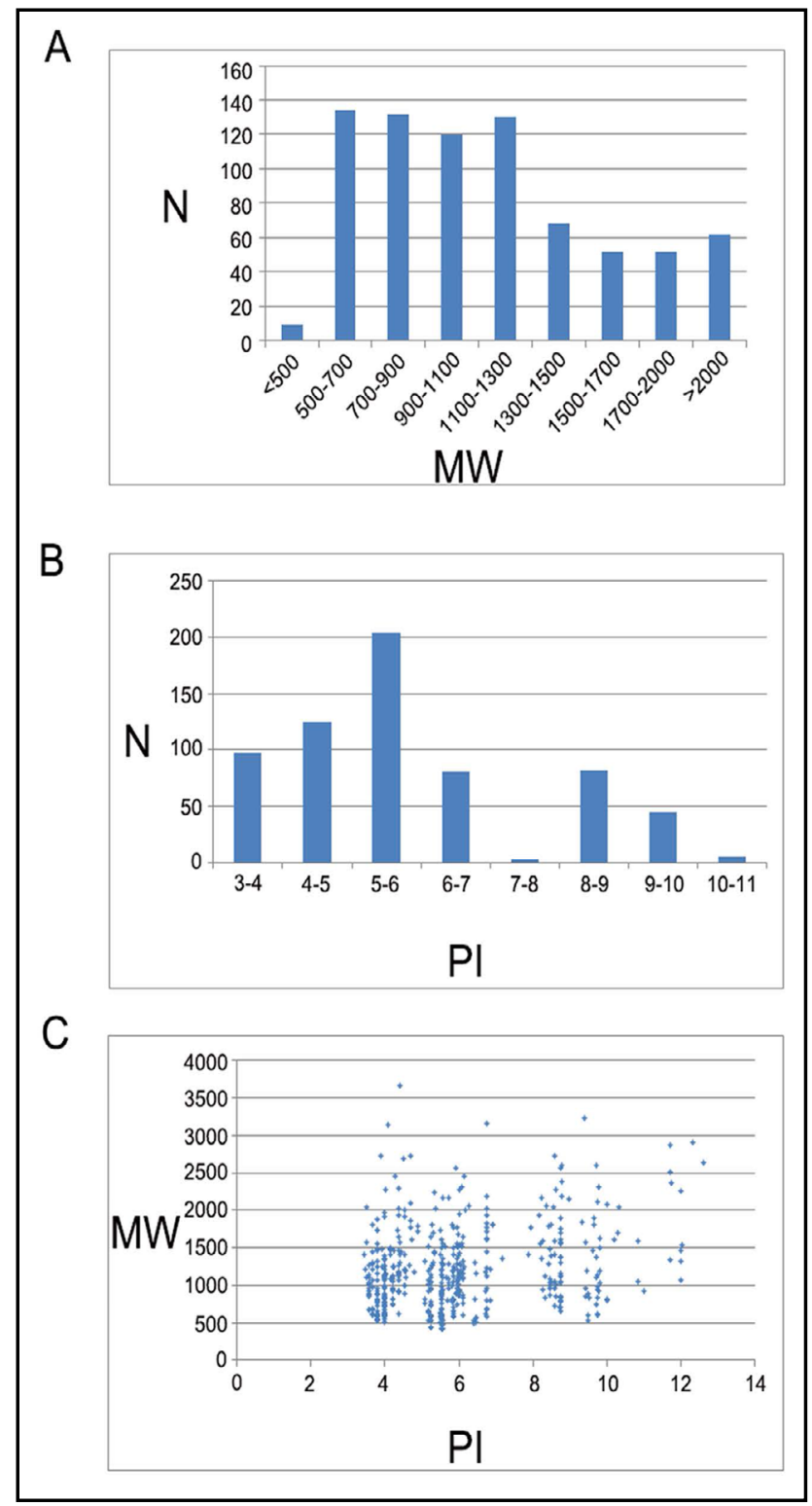

Fig. 1. Distributions of identified peptides. (A) Distribution of molecular weights (MW) of all peptides. (B) Distribution of isoelectric points (pI) of all peptides. (C) Scatter plot of MW versus $\mathrm{pI}$ distribution of the peptides. The data in all panels include all the peptides identified in both control and GDM sample groups. 
Fig. 2. Diseases, biological functions, and networks of precursors of identified peptides determined by IPA. Proteins in red are upregulated, proteins in green are downregulated, and color intensity is proportional to the magnitude of the expression change between control and GDM groups. The blue dotted line indicates suppression, the yellow dotted line reflects an inconsistency with downstream molecules, and the gray line signifies that no prediction was made. The dotted lines suggest an indirect relationship.

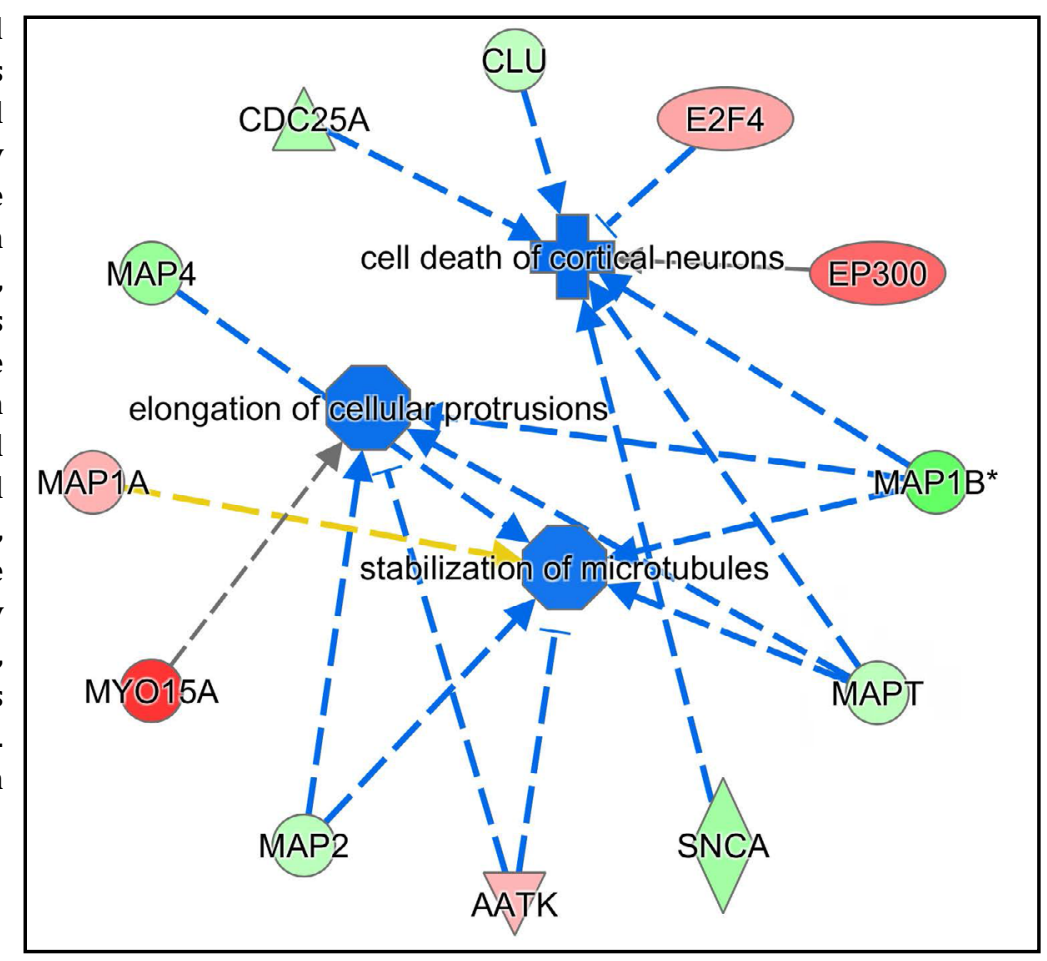

pathways were identified, of which FXR/RXR activation, the intrinsic prothrombin activation pathway, dendritic cell maturation, the ILK signaling pathway, and the AMPK signaling pathway all had a positive z-score (Suppl. Fig S1 - For all supplemental material see www. karger.com/10.1159/000495538/).

A total of 197 statistically significant disease categories were identified, the most prominent of which were cell death of cortical neurons, elongation of cellular protrusions, and stabilization of microtubules. We arranged 12 differentially expressed protein nodes around these three categories (Fig. 2).

IPA was also used to analyze the molecular networks associated with main disease category. The principal networks identified were lipid metabolism, molecular transport and small molecule biochemistry. Lipid metabolism consisted of six parts: concentration of sterol, transport of phospholipid, quantity of vitamin A, clearance of lipid, clearance of triacylglycerol, and efflux of phospholipid. Eleven of 13 proteins were associated with sterol concentration, four are associated with phospholipid transport, and three were associated with lipid clearance, clearance of triacylglycerol and efflux of phospholipid (Fig. 3).

\section{Putative biomarker peptides in the onset of GDM}

All differentially expressed peptides in glucose and fat metabolism were analyzed. For example, Table 2 presents the peptides whose precursor proteins were associated with the concentration of D-glucose, including the amino acid sequences, amino acids with PTM, peptide location, and domain/region description. Fig. 4 shows the network of these precursor proteins.

\section{Random validation of putative biomarker peptides using LC-MS/MS}

To validate the differential expression levels of the putative biomarker peptides, we collected serum samples from 30 normal pregnant women and 30 women with GDM (GDM group) at 16-18 weeks of gestation. Five peptides, which were dramatically highly expressed or exclusively expressed in the GDM group, were randomly chosen and synthesized. Four peptides were identified by LC-MS/MS, and their expression levels were consistent with those obtained in the primary results (Fig. 5, Table 4). 

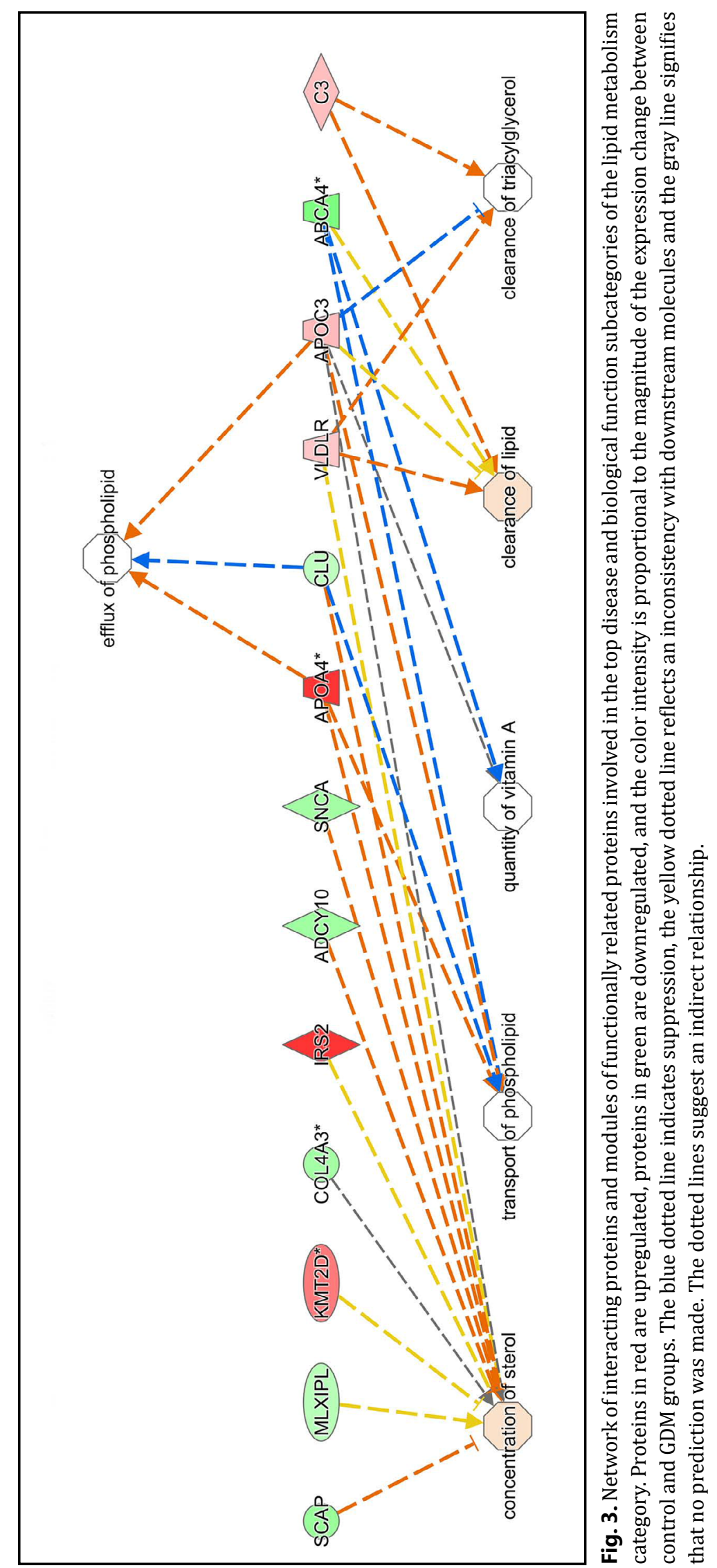


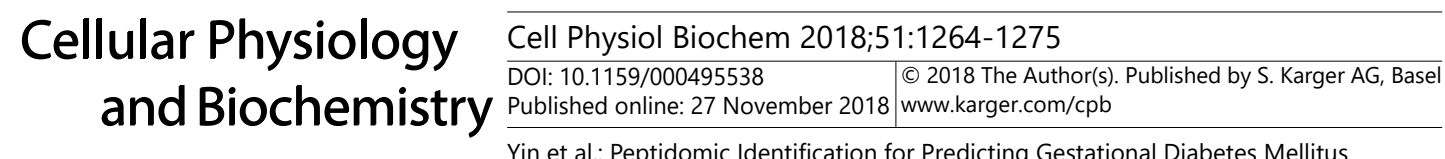

Table 2. Identified differentially expressed peptides within the functional domains of D-glucose-related proteins, and their post-translational modifications (PTM)

\begin{tabular}{lcccc}
\hline Precursor protein & Peptides & Peptide location & Doman/region description & $\begin{array}{c}\text { Fold change } \\
\text { (GDM/Control) }\end{array}$ \\
\hline APOA4 & DKTVSLPELEQQQEQQQEQQQ & $365-377$ & & \\
& EELRRSLAP & $217-225$ & AA approximate tandem repeats & 5.837 \\
C3 & KTVAVRTLDP & $940-949$ & & 1.821 \\
CAMKK2 & RPAPGG & $217-222$ & RP domain & -2.855 \\
EP300 & AALGLPYQVN & $468-478$ & & 4.330 \\
IRS2 & RTYSLTT & $574-580$ & & 5.801 \\
ITGB2 & KGYPI & $123-127$ & VWFA & -2.740 \\
MAGEL2 & ATATTQEASKTSVE & $864-877$ & & -2.054 \\
& LSESPGSSLPVVSEVASVSP & $981-1001$ & & 1.631 \\
MLXIPL & TAGSNNPCLTQ & $520-530$ & & -2.118 \\
PARG & KWLGTP & $452-457$ & & -2.735 \\
PKD1 & FPASPGATLVGP & $269-280$ & A-domain & -3.610 \\
& TNQTVLIRS & $2352-2360$ & WSC, PKD 1 & -3.152 \\
& EAATCAGPG & $151-159$ & REJ & 2.109 \\
RNF213 & EAGGCALNFGPRGSST & $2306-2322$ & LRRCT & 3.961 \\
& YLLVLTKN & $3041-3048$ & RING-type zinc finger domain & -3.971 \\
\hline
\end{tabular}

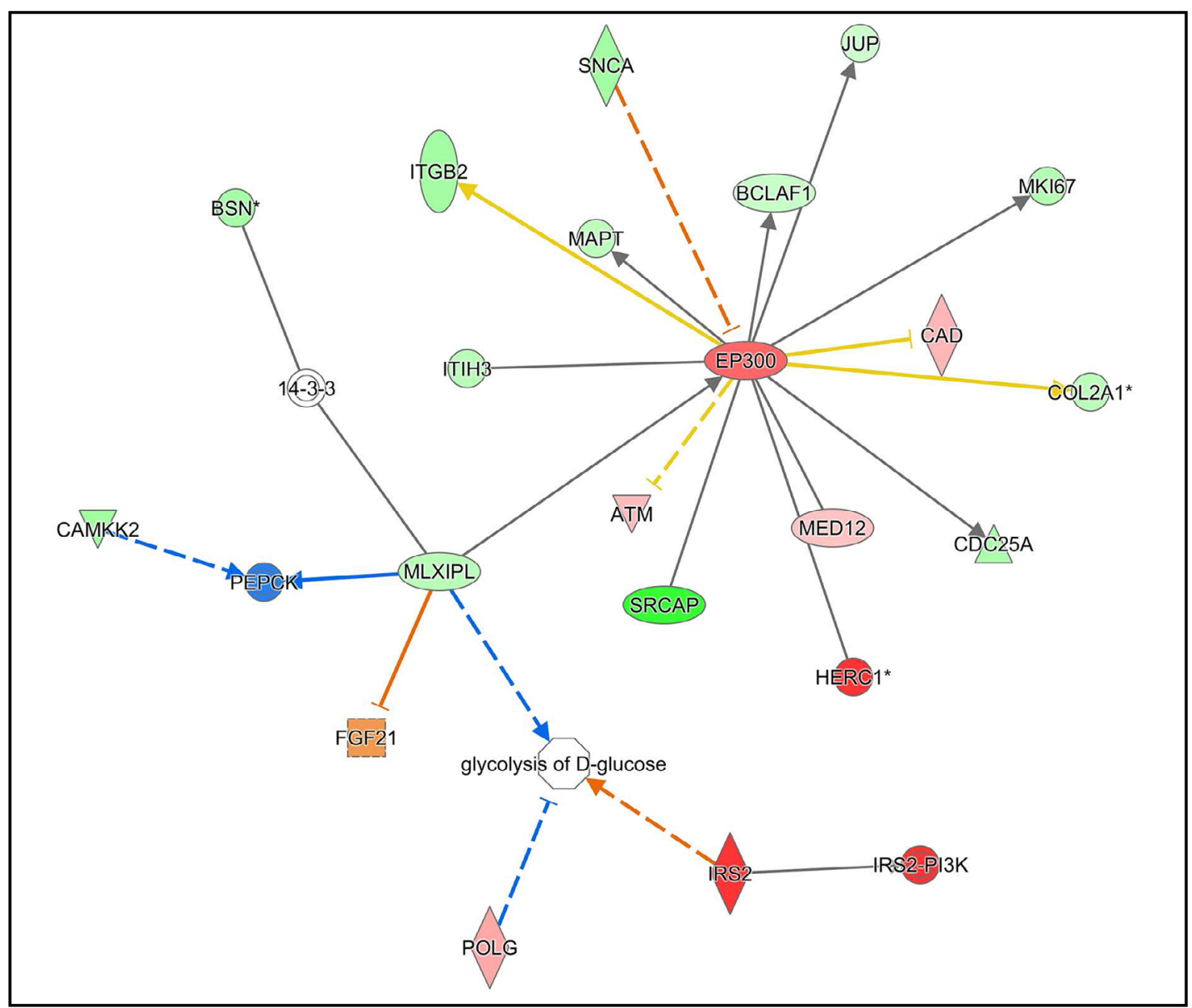

Fig. 4. Network of $\mathrm{D}$-glucose concentration. Red indicates upregulated proteins, green indicates downregulated proteins, and the color intensity is proportional to the magnitude of the expression change between the control and GDM groups. The blue dotted line indicates suppression, the yellow dotted line reflects an inconsistency with downstream molecules, and the gray line signifies that no prediction was made. Solid lines suggest a direct relationship, while the dotted lines suggest an indirect relationship. 

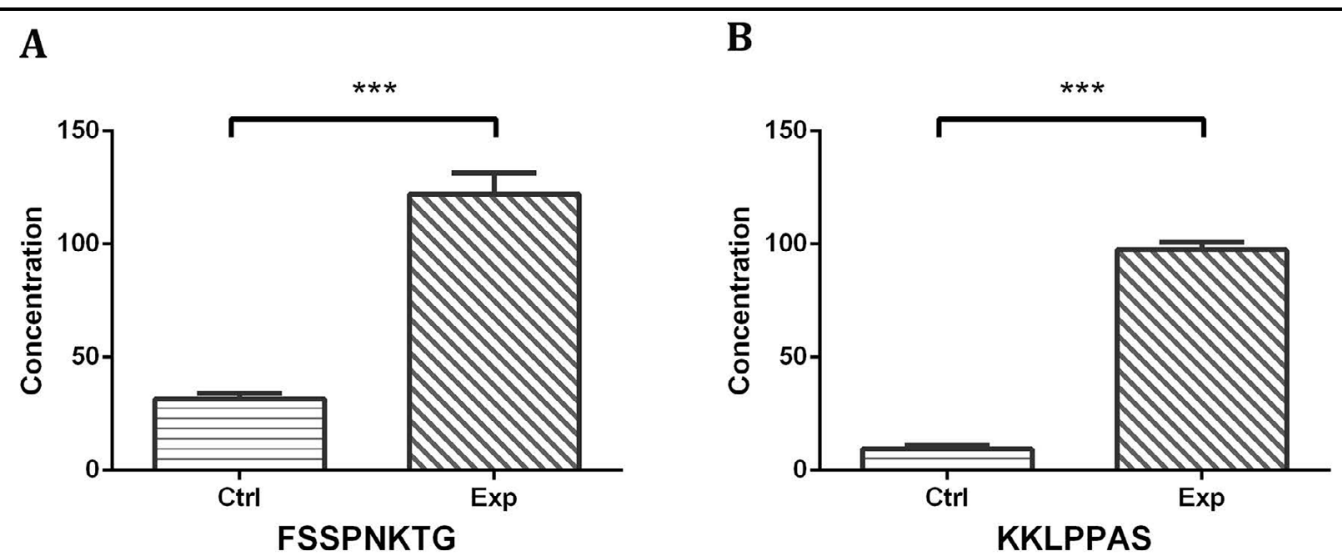

C

D
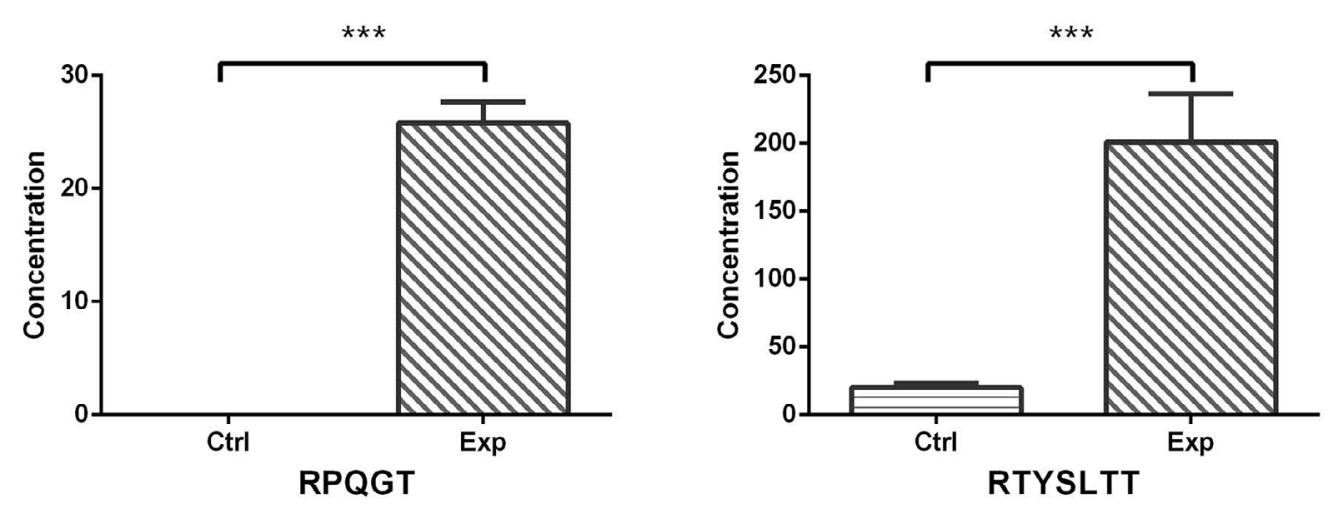

Fig. 5. Validation of four randomly selected peptides. Four peptides that were dramatically highly expressed or exclusively expressed in the GDM group were validated by LC-MS/MS using serum samples from 30 normal controls and 30 GDM women at 16-18 weeks of gestation. A: FSSPNKTG, B: KKLPPAS, C: RPQGT, D: RTYSLTT

\section{Identification of candidate enzymes}

We selected "RTYSLTT," which showed a 10.112-fold higher change in the GDM group compared to the control group, as our target. A search of the peptidase database (MEROPS) identified a number of candidate peptidases that would likely cleave the start and end bonds, also referred to as the K-RT and T-P sites of RTYSLTT (Table 3). This list included peptidases and proteases from different classes (cysteine, aspartic, metallo, serine, and threonine), as well as soluble and membrane-bound forms of the enzyme. According to this list, cathepsin $\mathrm{V}$ and cathepsin L could cleave RTYSLTT at both the cleavage sites, suggesting that RTYSLTT may be regulated by a single enzyme. 


\section{Cellular Physiology Cell Physiol Biochem 2018;51:1264-1275

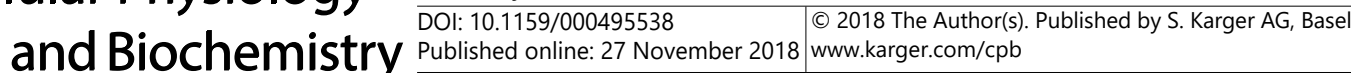 \\ Yin et al.: Peptidomic Identification for Predicting Gestational Diabetes Mellitus}

\section{Discussion}

Human serum is a rich source of endogenous peptides, many of which may serve as biomarkers. However, relatively few studies have explored the potential of this class of molecules as a source of GDM biomarkers. In this study, we focused on early detection and identification of alterations in peptide profiles between GDM patients and healthy controls.

Gestational diabetes mellitus (GDM) affects an increasing number of pregnant women worldwide. GDM is related to obesity, family history, ethnicity, and environmental factors. Poorly controlled glucose results in adverse outcomes for both mother and fetus. Therefore, early screening and diagnosing at appropriate gestational age is important to minimize adverse outcomes. Routine screening at 24-28 weeks with a glucose challenge test leaves limited time for intervention and prevention. There is evidence that many women manifest hyperglycemia early in pregnancy as a consequence of previously undiagnosed diabetes, or they display higher levels of insulin resistance and reduced capacity to increase insulin secretion, resulting in early decompensation as early onset GDM [17]. Recently several investigators reported early predictors of GDM may include high-sensitivity C-reactive protein [18], sex hormone binding globulin [19], soluble pro-renin receptor [20], glycosylated fibronectin [21] and galanin [22]. However, due to various limitations, these tests are not widely used. Using effective biomarkers for early prediction and screening of GDM, allows detection and treatment in a timely manner.

Genes and proteomics have been studied for years in the search for biological markers for a variety of diseases. However, due to the character of genes and proteomics that are not routinely secreted into biofluids [4], biomarker discovery has proved difficult. Peptidomics, low molecular weight (LMW) peptides and proteolytic fragments of molecular mass [23], are frequently secreted into serum. Therefore, peptidomics is becoming an intense focus of research on serum biomarkers. In these studies, protein mixtures are digested with trypsin, and the resulting peptide mixtures are analyzed by tandem mass spectrometry (LC-MS/MS). Today, many peptides have been found to biological markers for the cancer. For example, plasma fibronectin 1 (FN1) might be a tool for screening and diagnosis of renal cell carcinoma, as plasma levels of FN1 are up-regulated in the early stage of RCC [24].

Table 3. Peptide "RTYSLTT"-degrading enzymes predicted by MEROPS. Cathepsin V and cathepsin L (in bold) were the only enzymes predicted to cleave both "RTYSLTT" cleavage sites

\begin{tabular}{lc}
\hline Peptidase & Enzyme class \\
\hline K-RT cleavage site & \\
Cathepsin V & Cysteine \\
Cathepsin L & Cysteine \\
Cathepsin K & Cysteine \\
Trypsin 1 & Serine \\
& \\
T-P cleavage site & \\
Polyporopepsin & \\
Necepsin-1 & Aspartic \\
Necepsin-2 & Aspartic \\
Cathepsin V & Aspartic \\
Cathepsin L & Cysteine \\
Cathepsin S & Cysteine \\
Falcipain-3 & Cysteine \\
Cathepsin L3 & Cysteine \\
Calpain-1 & Cysteine \\
Calpain-2 & Cysteine \\
Legumain, animal-type & Cysteine \\
Hedgehog protein & Cysteine \\
Matrix metallopeptidase-9 & Cysteine \\
Matrix metallopeptidase-3 & Metallo \\
Matrix metallopeptidase-6 & Metallo \\
Endothelin-converting enzyme 1 & Metallo \\
Aminopeptidase P & Metallo \\
Pro-Hyp dipeptidase & Metallo \\
Kallikrein-related peptidase 6 & Metallo \\
Glutamyl endopeptidase I & Serine \\
Lactocepin-3 & Threonine \\
Keratinase & Threonine \\
Proteasome subunit beta 5c & \\
20 S Constitutive proteasome peptidase complex \\
26 S Proteasome peptidase complex & \\
\hline & \\
& \\
&
\end{tabular}

Table 4. Information of four peptides and precursor proteins (G/C: GDM/Control)

\begin{tabular}{ccccc}
\hline Accession & Protein & Peptide & Fold change $(\mathrm{G} / \mathrm{C})$ & P value \\
\hline P35658 & NU214 & FSSPNKTG & 3.869 & $<0.001$ \\
Q6PFW1 & VIP1 & KKLPPAS & 10.273 & $<0.001$ \\
P32004 & L1CAM & RPQGT & \#DIV/0! & $<0.001$ \\
Q9Y4H2 & IRS2 & RTYSLTT & 10.112 & $<0.001$ \\
\hline
\end{tabular}




\section{Cellular Physiology Cell Physiol Biochem 2018;51:1264-1275

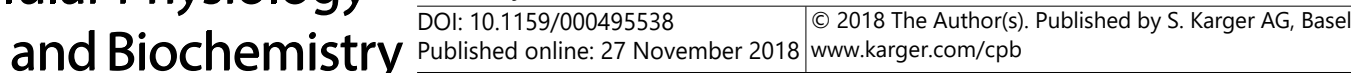 \\ Yin et al.: Peptidomic Identification for Predicting Gestational Diabetes Mellitus}

Peptides are stable cleavage products generated from protein degradation by proteolysis [25]. It has been reported that peptidomic analyses of body fluids yielded disease biomarkers representing cleavage fragments [26]. Different cleavage sites may represent different protease activities, as the specificity of a protease is reflected by the cleavage events it triggers. We found that the proteases cathepsin $\mathrm{V}$ and cathepsin $\mathrm{L}$ can cleave RTYSLTT at both cleavage sites. Cathepsin $\mathrm{V}$ has been reported to be associated with type 1 diabetes [27], and cathepsin L activity can control adipogenesis and glucose tolerance [28].

One of the major challenges in peptidome research is processing and analyzing the large amount of information generated from a particular biological context. In our study, the peptidome was expanded to identify the precursor proteins, which could reveal important information regarding glucose and fat metabolism. For example, the peptide RTYSLTT is a fragment of insulin receptor substrate 2 (IRS-2), whose expression in pancreatic $\beta$-cells is essential to regulation of $\beta$-cell metabolism and survival. When IRS- 2 expression is specifically increased in $\beta$-cells, it maintains adequate functional $\beta$-cell mass, and thereby forestalls the onset of diabetes [29]. IRS-2 depends on the downstream IRS/PI3-kinase/Akt signaling cascade required for insulin regulation in glucose homeostasis [30]. It has been suggested that IRS-2 plays an important role in glucose homeostasis, particularly in the liver [31]. Araki found that loss of IRS-2 causes diabetes in mice due to $\beta$-cell insufficiency and peripheral insulin resistance [32]. GDM is the consequence of the imbalanced insulin secretion that occurs to counteract insulin resistance. Previous studies in insulin-sensitive maternal peripheral tissues have showed altered expression of important proteins involved in insulin signaling in GDM. In skeletal muscle, the IRS-2 protein was more highly expressed and its phosphorylation was dramatically reduced in women with GDM compared with pregnant controls, leading to an alteration in the insulin signaling pathway. The expression of IRS-2 in adipose tissue was increased in women with GDM compared with the controls [33]. Some investigators found a correlation between IRS-2 expression and maternal insulin levels. Fetal insulin had a weak correlation with IRS-2 [34]. In our study, we found that RTYSLTT expression was substantially higher in the GDM group (fold change $=10.112$ ) than in the control group. Whether this peptide was derived from IRS-2 degradation or from newly synthesized material, its differential abundance in early second-trimester serum of GDM women may reflect changes in the expression and function of IRS-2, and consequently be putative bioactive peptides for GDM.

PKD1 or polycystin-1 has been demonstrated to play an essential role in renal tubular morphogenesis. Disruption of its function causes cystogenesis in human autosomal-dominant polycystic kidney disease (ADPKD) [35]. Currently, however, there is no research on the role of PKD1 in diabetes. In the present study, we found four differentially expressed endogenous peptides derived from PKD1 mapped to the functional domains of this protein (Table 2). It is known that cleavage of precursor proteins by proteases generates peptides that may gain specialized functions not ascribed to their precursors, which could reflect the state of a cell under certain physiological conditions or pathological processes [36]. Therefore, it is reasonable to believe that although the functions of these PKD1-derived peptides may not have a strong association with their precursor protein, these peptides may play an important role in the early prediction of GDM.

Our results are the first peptidome profile of the differentially expressed peptides in maternal serum at 16-18 weeks of gestation, which was much earlier than the time of GDM onset. Therefore, a large amount of differentially expressed peptides identified in this study may not be the result of GDM but its cause or concomitant factors involved in its progression. Furthermore, our study provided a background for future research; impaired glucose tolerance and GDM remain a public health priority, and earlier pregnancy identification of at-risk women has the potential to increase the time for intervention and prevention. 


\section{Cellular Physiology Cell Physiol Biochem 2018;51:1264-1275

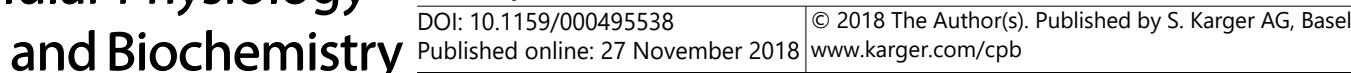 \\ Yin et al.: Peptidomic Identification for Predicting Gestational Diabetes Mellitus}

\section{Acknowledgements}

This work was financially supported by the National Natural Science Foundation of China (81571458, 81100436), Nanjing Health Science and Technology Development Fund (YKK14126, JQX1501), State Key Laboratory of Reproductive Medicine Project (SKLRM2015B2), Nanjing Health Young Talent project (JQX1501), National Key Research and Development Program of China (2016YFC1000300, 2016YFC1000303), Nanjing high-level talent training project (333 project) and Maternal and Key Personnel of Maternal and Child Health Project in Jiangsu Province. Technology Development Foundation of Jiangsu Provincial Commission of Health Family Planning (H201343), Technology Development Foundation of Nanjing (ZKX1401, 201405041).

\section{Disclosure Statement}

The authors have declared no conflicts of interest.

\section{References}

1 Committee on Practice Bulletins-Obstetrics: ACOG Practice Bulletin No. 190: Gestational Diabetes Mellitus. Obstet Gynecol 2018;131:e49-e64.

-2 Schneider S, Bock C, Wetzel M, Maul H, Loerbroks A: The prevalence of gestational diabetes in advanced economies. J Perinat Med 2012;40:511-520.

-3 Ferrara A: Increasing prevalence of gestational diabetes mellitus: a public health perspective. Diabetes Care 2007;30:S141-S146.

74 American Diabetes Association: Standards of medical care in diabetes-2014. Diabetes Care 2014;37:S14-S80.

5 Bentley-Lewis R, Powe C, Ankers E, Wenger J, Ecker J, Thadhani R: Effect of race/ethnicity on hypertension risk subsequent to gestational diabetes mellitus. Am J Cardiol 2014;113:1364-1370.

6 Mitanchez D: Foetal and neonatal complications in gestational diabetes: perinatal mortality, congenital malformations, macrosomia, shoulder dystocia, birth injuries, neonatal complications. Diabetes Metab 2010;36:617-627.

7 Eleftheriades M, Papastefanou I, Lambrinoudaki I, Kappou D, Lavranos D, Akalestos A, Souka AP, Pervanidou P, Hassiakos D, Chrousos GP: Elevated placental growth factor concentrations at 11-14 weeks of gestation to predict gestational diabetes mellitus. Metabolism 2014;63:1419-1425.

8 Syngelaki A, Kotecha R, Pastides A, Wright A, Nicolaides KH: First-trimester biochemical markers of placentation in screening for gestational diabetes mellitus. Metabolism 2015;64:1485-1489.

-9 de Seymour JV, Conlon CA, Sulek K, Villas Bôas SG, McCowan LM, Kenny LC, Baker PN: Early pregnancy metabolite profiling discovers a potential biomarker for the subsequent development of gestational diabetes mellitus. Acta Diabetol 2014;(5):887-890.

$>10$ Ivanov VT, Yatskin ON: Peptidomics: a logical sequel to proteomics. Expert Rev Proteomics 2005;2:463473.

11 Schulz-Knappe P, Zucht HD, Heine G, Jürgens M, Hess R, Schrader M: Peptidomics: the comprehensive analysis of peptides in complex biological mixtures. Comb Chem High Throughput Screen 2001;4:207-217.

12 American Diabetes Association: Diagnosis and classification of diabetes mellitus. Diabetes Care 2011;34:S62-S69.

13 Huang Z, Zhang S, Hang W, Chen Y, Zheng J, Li W, Xing J, Zhang J, Zhu E, Yan X: Liquid chromatography-mass spectrometry based serum peptidomic approach for renal clear cell carcinoma diagnosis. J Pharm Biomed Anal 2014;100:175-183.

14 Lin H, He L, Ma B: A combinatorial approach to the peptide feature matching problem for label-free quantification. Bioinformatics 2013;29:1768-1775.

15 Bortner JD Jr, Das A, Umstead TM, Freeman WM, Somiari R, Aliaga C: Phelps DS, El-Bayoumy K. Downregulation of 14-3-3 isoforms and annexin A5 proteins in lung adenocarcinoma induced by the tobaccospecific nitrosamine NNK in the A/J mouse revealed by proteomic analysis. J Proteome Res 2009;8:40504061. 


\section{Cellular Physiology Cell Physiol Biochem 2018;51:1264-1275 \begin{tabular}{l|l|l} 
and Biochemistry Published online: 27 November 2018 & $\begin{array}{l}\text { ○ } 2018 \text { The Author(s). Published by S. Karger AG, Basel } \\
\text { www.karger.com/cpb }\end{array}$ \\
\hline
\end{tabular}

16 Rawlings ND, Barrett AJ, Finn R: Twenty years of the MEROPS database of proteolytic enzymes, their substrates and inhibitors. Nucleic Acids Res 2016;44:D343-D350.

17 Seshiah V, Balaji V, Balaji MS, Paneerselvam A, Arthi T, Thamizharasi M, Datta M. Gestational diabetes mellitus manifests in all trimesters of pregnancy. Diabetes Res Clin Pract 2007;77:482-484.

$>18$ Ozgu-Erdinc, AS, Yilmaz S, Yeral MI, Seckin KD, Erkaya S, Danisman AN: Prediction of gestational diabetes mellitus in the first trimester: comparison of C-reactive protein, fasting plasma glucose, insulin and insulin sensitivity indices. J Matern Fetal Neonatal Med 2015;28:1957-1962.

19 Caglar GS, Ozdemir ED, Cengiz SD, Demirtaş S: Sex-hormone-binding globulin early in pregnancy for the prediction of severe gestational diabetes mellitus and related complications. J Obstet Gynaecol Res 2012;38:1286-1293.

20 Watanabe N, Morimoto S, Fujiwara T, Suzuki T, Taniguchi K, Mori F, Ando T, Watanabe D, Kimura T, Sago $\mathrm{H}$, Ichihara A: Prediction of gestational diabetes mellitus by soluble (pro)renin receptor during the first trimester. J Clin Endocrinol Metab 2013;98:2528-2535.

21 Rasanen, J, Girsen A, Lu X, Lapidus JA, Standley M, Reddy A, Dasari S, Thomas A, Jacob T, Pouta A, Surcel HM, Tolosa JE, Gravett MG, Nagalla SR: Comprehensive maternal serum proteomic profiles of preclinical and clinical preeclampsia. J Proteome Res 2010;9:4274-4281.

22 Fang P, Bo P, Shi M, Yu M, Zhang Z: Circulating galanin levels are increased in patients with gestational diabetes mellitus. Clin Biochem 2013;46:831-833.

-23 Tirumalai RS, Chan KC, Prieto DA, Issaq HJ, Conrads TP, Veenstra TD: Characterization of the low molecular weight human serum proteome. Mol Cell Proteomics 2003;2:1096-1103.

24 Yokomizo A, Takakura M, Kanai Y, Sakuma T, Matsubara J, Honda K, Naito S, Yamada T, Ono M: Use of quantitative shotgun proteomics to identify fibronectin 1 as a potential plasma biomarker for clear cell carcinoma of the kidney. Cancer Biomark 2011;10:175-183.

25 Shahinian H, Tholen S, Schilling 0: Proteomic identification of protease cleavage sites: cell-biological and biomedical applications. Expert Rev Proteomics 2013;10:421-433.

-26 Villanueva J, Shaffer DR, Philip J, Chaparro CA, Erdjument-Bromage H, Olshen AB, Fleisher M, Lilja H, Brogi E, Boyd J, Sanchez-Carbayo M, Holland EC, Cordon-Cardo C, Scher HI, Tempst P: Differential exoprotease activities confer tumor-specific serum peptidome patterns. J Clin Invest 2006;116:271-284.

-27 Viken MK, Sollid HD, Joner G, Dahl-Jørgensen K, Rønningen KS, Undlien DE, Flatø B, Selvaag AM, Førre Ø, Kvien TK, Thorsby E, Melms A, Tolosa E, Lie BA: Polymorphisms in the cathepsin L2 (CTSL2) gene show association with type 1 diabetes and early-onset myasthenia gravis. Hum Immunol 2007;68:748-755.

28 Yang M, Zhang Y, Pan J, Sun J, Liu J, Libby P, Sukhova GK, Doria A, Katunuma N, Peroni OD, Guerre-Millo M, Kahn BB, Clement K, Shi GP: Cathepsin L activity controls adipogenesis and glucose tolerance. Nat Cell Biol 2007;9:970-977.

29 Hennige AM, Burks DJ, Ozcan U, Kulkarni RN, Ye J, Park S, Schubert M, Fisher TL, Dow MA, Leshan R, Zakaria M, Mossa-Basha M, White MF: Upregulation of insulin receptor substrate-2 in pancreatic beta cells prevents diabetes. J Clin Invest 2003;112:1521-1532.

-30 Zhu N, Zhang D, Xie H, Zhou Z, Chen H, Hu T, Bai Y, Shen Y, Yuan W, Jing Q, Qin Y: Endothelial-specific intronderived miR-126 is down-regulated in human breast cancer and targets both VEGFA and PIK3R2 Mol Cell Biochem 2011;351:157-164.

31 Burks DJ, White MF: IRS proteins and beta-cell function. Diabetes 2001;50:S140-S145.

-32 Araki E, Lipes MA, Patti ME, Brüning JC, Haag B 3rd, Johnson RS, Kahn CR: Alternative pathway of insulin signalling in mice with targeted disruption of the IRS-1 gene. Nature 1994;372:186-190.

-33 Colomiere M, Permezel M, Lappas M: Diabetes and obesity during pregnancy alter insulin signalling and glucose transporter expression in maternal skeletal muscle and subcutaneous adipose tissue. J Mol Endocrinol 2010;44:213-223.

34 Colomiere M, Permezel M, Riley C, Desoye G, Lappas M: Defective insulin signaling in placenta from pregnancies complicated by gestational diabetes mellitus. Eur J Endocrinol 2009;160:567-578.

35 Qian F, Boletta A, Bhunia AK, Xu H, Liu L, Ahrabi AK, Watnick TJ, Zhou F, Germino GG: Cleavage of polycystin-1 requires the receptor for egg jelly domain and is disrupted by human autosomal-dominant polycystic kidney disease 1-associated mutations. Proc Natl Acad Sci U S A 2002;99:16981-16986.

-36 Hussain M, Awan FR: Hypertension regulating angiotensin peptides in the pathobiology of cardiovascular disease. Clin Exp Hypertens 2018;40:344-352. 